\title{
Survival from acute non-lymphocytic leukaemia, 1971-88: a population based study
}

\author{
C A Stiller, E M Eatock
}

\begin{abstract}
Survival rates were studied among 1258 children with acute non-lymphocytic leukaemia diagnosed in 1971-88 and included in the population based National Registry of Childhood Tumours. Of the total, $147(12 \%)$ died without receiving treatment. Among the remaining treated children, actuarial five year survival rates were $6 \%$ in $1971-4,15 \%$ in $1975-9,23 \%$ in $1980-3$, and $40 \%$ in 1984-8. Infants aged less than 1 year had a significantly worse prognosis and there was a significant trend towards lower survival rates with increasing white cell count. No independent significant effects on survival were found with sex, French-American-British (FAB) subtype, or the presence or absence of Down's syndrome. Children entered in national trials had a higher survival rate than those who were not entered, and children treated at teaching hospitals had a higher survival rate than those who were treated elsewhere. Among the 535 $(43 \%)$ children who survived at least one year from diagnosis no factor studied had a significant effect on survival, emphasising the importance of achieving first remission as a determinant of long term survival.
\end{abstract}

(Arch Dis Child 1994; 70: 219-223)

The outlook for children with acute nonlymphocytic leukaemia (ANLL) diagnosed during the early 1970s was bleak, with fewer than $5 \%$ surviving for five years, but by the mid-1980s five year survival rates in Britain had improved to over $25 \% .^{1}$ Throughout this period the Medical Research Council (MRC) organised a succession of clinical trials for the treatment of ANLL. ${ }^{2-6}$ These were all open to children, though only one, the UK Acute Myeloid Leukaemia (UKAML) trial which ran from the end of 1974 to early 1980 , was specifically set up for children ${ }^{5}$ and the patients in the other trials were predominantly adults. Substantial numbers of children were also entered in a non-MRC multicentre study in the 1980 s. $^{7}$ From 1971 to 1984 children with acute lymphoblastic leukaemia had a higher survival rate if they were patients at hospitals which treated larger numbers of children with this disease, or if they were entered in the MRC trials. ${ }^{8}$ Children with ANLL diagnosed from 1977 to 1984 who were treated at a teaching hospital had a higher survival rate than those treated elsewhere. ${ }^{9}$

The purpose of this study was to investigate possible prognostic factors, including those related to patterns of medical care, for ANLL in childhood in a large population based series and to document survival rates since 1985 .

\section{Patients and methods}

The population based National Registry of Childhood Tumours ascertains cases of malignant disease in children under 15 years of age from the national cancer registration systems of England, Scotland, and Wales, specialist children's tumour registries in several regions, notifications to the MRC's leukaemia trials, the UK Children's Cancer Study Group (CCSG), and death certificates.

Confirmation of diagnosis and information on congenital abnormalities, treatment, and follow up are obtained from the hospitals at which the children were treated, from their family doctors, and from clinical trial records. Children not already known to have died are flagged in the NHS central registers so that any further deaths are automatically notified to us.

Cases ascertained from death certificates alone are not included in analyses of survival. From all other sources combined, there were 1258 children domiciled in Great Britain with ANLL (including acute monocytic leukaemia, erythroleukaemia, and megakaryocytic leukaemia) diagnosed in the period 1971-88.

For children who survived to be treated the treatment centre was defined as the hospital from which chemotherapy was directed. Where a hospital closed and treatment of childhood leukaemia was transferred to another hospital, or where treatment was administered by a team of clinicians working jointly at two hospitals, the treatment centre was taken to include both hospitals.

Follow up was complete to the end of 1992 for all but four of the survivors. Survival rates were analysed by standard methods to examine the effects of age, sex, morphological subgroups defined by the French-AmericanBritish (FAB) classification, ${ }^{10}$ white cell count, inclusion in trials, and type of treatment centre. Calendar periods of diagnosis were defined as 1971-4, 1975-9, 1980-3, and $1984-8$, to coincide approximately with the periods of accrual to successive national trials. Survival rates were also analysed for all children who survived at least three months to investigate whether significant prognostic factors for the entire series were still important when patients who had probably not entered remission were excluded; exclusion of children who died within three months should also eliminate possible bias if patients who were perceived to have a poor prognosis were
Correspondence to: Dr Stiller.

Accepted 27 October 1993 
Table 1 Five year actuarial survival rates and proportions of treated children in successive calendar periods

\begin{tabular}{lcccc}
\hline & $\begin{array}{c}1971-4 \\
(n=332)\end{array}$ & $\begin{array}{c}1975-9 \\
(n=394)\end{array}$ & $\begin{array}{c}1980-3 \\
(n=219)\end{array}$ & $\begin{array}{c}1984-8 \\
(n=313)\end{array}$ \\
\hline $\begin{array}{l}\text { Five year survival rate (\%) } \\
\text { No (\%) of children treated }\end{array}$ & 5 & 13 & 20 & 36 \\
Five year survival rate of treated children (\%) & $293(88)$ & $347(88)$ & $188(86)$ & $283(90)$ \\
& 6 & 15 & 23 & 40 \\
\hline
\end{tabular}

Table 2 No (\%) of children with different FAB subtypes first treated in 1980-8

\begin{tabular}{llllllllll}
\hline $\begin{array}{l}\text { Year of } \\
\text { diagnosis }\end{array}$ & $M 1$ & $M 2$ & $M 3$ & $M 4$ & $M 5$ & $M 6$ & $M 7$ & Unknown & Total \\
\cline { 2 - 8 } & $M 1(5)$ & $32(17)$ & $12(6)$ & $30(16)$ & $22(12)$ & $12(6)$ & $8(4)$ & $63(34)$ & $188(100)$ \\
$1980-3$ & $98(10)$ & $58(20)$ & $16(6)$ & $66(23)$ & $34(12)$ & $10(4)$ & $17(6)$ & $54(19)$ & $283(100)$ \\
$1984-8$ & 28 &
\end{tabular}

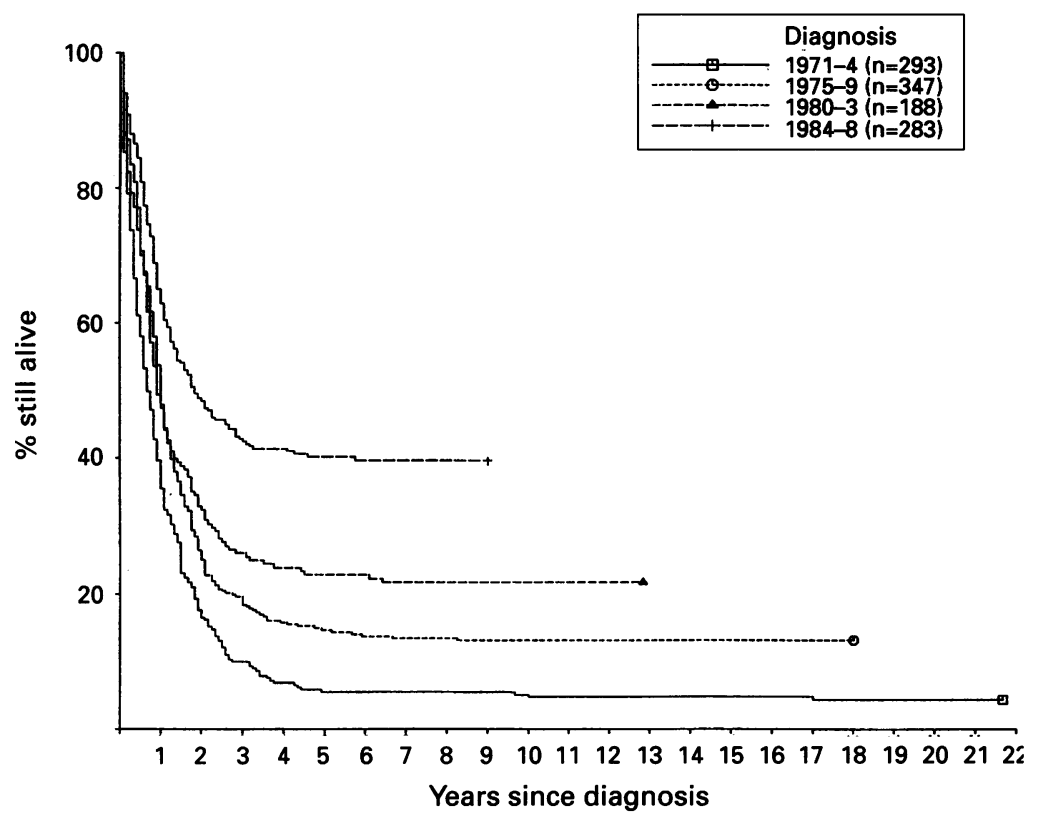

Figure 1 Actuarial survival curves for children first treated during the period 1971-88 classified by period of diagnosis.

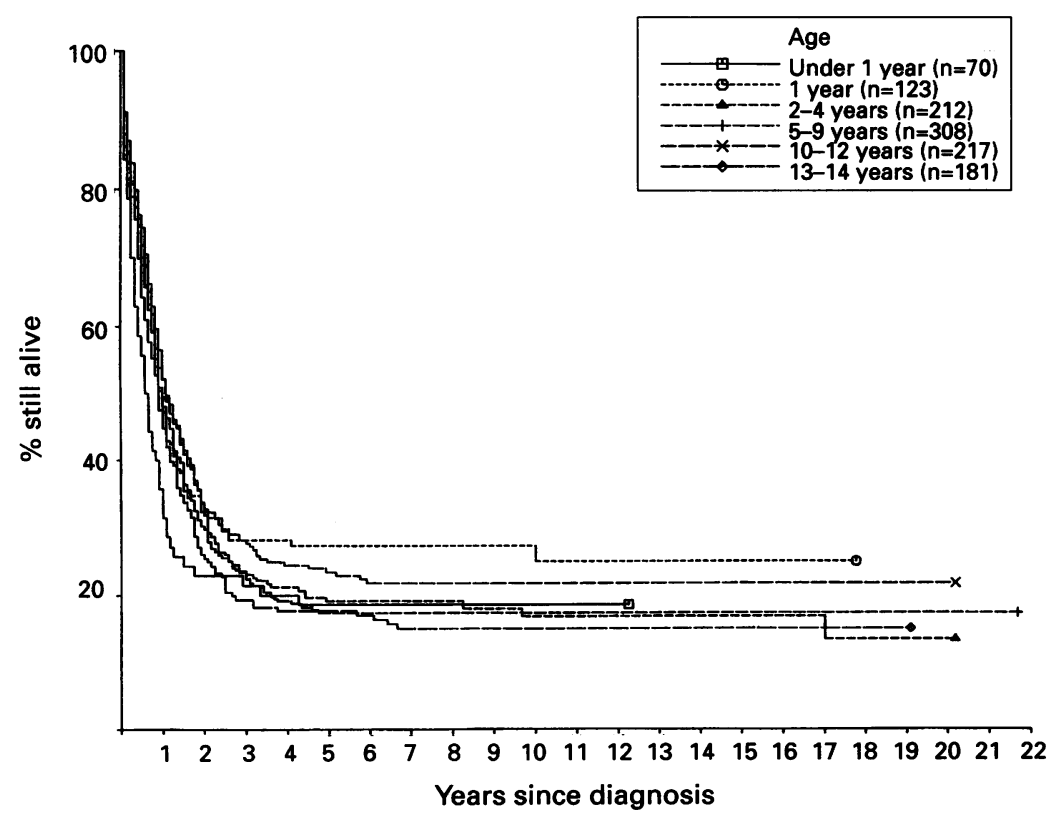

Figure 2 Actuarial survival curves for children first treated during the period 1971-88 classified by age at diagnosis. selectively not entered in trials or referred to teaching hospitals. Survival rates for one year survivors were analysed to study factors influencing survival when patients who had relapsed relatively early were also excluded.

\section{Results}

Table 1 shows the actuarial five year survival rates for successive calendar periods of diagnosis. Of the 1258 children in the study, $147(12 \%)$ died without receiving treatment for their leukaemia. There was little sign of any change in the proportion of untreated children with the period of diagnosis. The proportion of untreated children was higher when ANLL occurred with Down's syndrome. Among the 72 children with Down's syndrome in the study, $44(61 \%)$ were untreated; these included $13(87 \%)$ of the 15 Down's syndrome children who also had cardiac abnormalities and $13(93 \%)$ of the 14 who were aged less than 1 year at diagnosis. The proportion of non-Down's syndrome children who were untreated also decreased with increasing age at diagnosis from $32 \%(33 / 102)$ at age 0 to $10 \%$ $(12 / 122)$ at age 1 year and $6 \%(58 / 962)$ at age 2-14 years. There were only three non-Down's syndrome children recorded as having cardiac abnormalities and all three received treatment for their leukaemia.

The other analyses are based on the 1111 children who received treatment for ANLL. There was a highly significant trend in survival with period of diagnosis (fig 1: $\chi^{2}=110 \cdot 3$ on $1 \mathrm{df}, \mathrm{p}<0.0001$ ), the five year survival rate increasing from $6 \%$ for children first treated in 1971-4 to $40 \%$ for $1984-8$. All subsequent analyses allow for the period of diagnosis.

Among treated patients there was no significant difference in survival rates between the sexes. Children with Down's syndrome who received treatment for their leukaemia had a similar survival rate to non-Down's syndrome children. There was a significant variation in survival with age at diagnosis (fig $2: \chi^{2}=12 \cdot 7$ on $5 \mathrm{df}, \mathrm{p}<0.05$ ); this was largely due to a much lower rate among infants aged less than 1 year $\left(\chi^{2}=9.98\right.$ on $\left.1 \mathrm{df}, \mathrm{p}<0.01\right)$, particularly during the first year after diagnosis, though their long term survival was similar to that of older children.

There was a highly significant trend towards lower survival rates for children with a higher white cell count (fig $3: \chi^{2}=17 \cdot 5$ on $1 \mathrm{df}$, $\mathrm{p}<0.0001)$. Where possible the morphology of leukaemic cells was classified from available records according to the FAB system. Overall only $556(50 \%)$ treated patients could be classified, but the proportion increased to $66 \%$ for $1980-3$ and $81 \%$ for $1984-8$. Table 2 shows the FAB classification for children first treated in 1980-8. There was no significant difference in survival rates between subtypes during this period. Age, white cell count, and morphology were, however, interrelated: infants aged less than 1 year were less likely to have M2 leukaemia, children with M2 acute myeloid leukaemia were more likely to have a lower white cell count, and those with M4 
(myelomonocytic) leukaemia were more likely to have a higher count. A combined analysis of age, white cell count and FAB subtype was therefore carried out for children diagnosed in 1980-8, the effects of each factor being analysed while allowing for the other two. The

Table 3 No (\%) of children first treated in successive calendar periods by type of treatment centre

\begin{tabular}{lcccc}
\hline & $\begin{array}{l}1971-4 \\
(n=293)\end{array}$ & $\begin{array}{r}1975-9 \\
(n=347)\end{array}$ & $\begin{array}{r}1980-3 \\
(n=188)\end{array}$ & $\begin{array}{c}1984-8 \\
(n=283)\end{array}$ \\
\hline Teaching hospital & $179(61)$ & $286(82)$ & $164(87)$ & $261(92)$ \\
Other hospital & $114(39)$ & $61(18)$ & $24(13)$ & $22(8)$ \\
\hline
\end{tabular}

Table 4 No (\%) of children first treated in successive calendar periods who were entered in national trials, grouped by type of treatment centre

\begin{tabular}{lcccc}
\hline & $1971-4$ & $1975-9$ & $1980-3$ & $1984-8$ \\
\hline Teaching hospital & $20 / 179(11)$ & $138 / 286(48)$ & $86 / 164(52)$ & $120 / 261(46)$ \\
Other hospital & $7 / 114(6)$. & $13 / 61(21)$ & $7 / 24(29)$ & $5 / 22(23)$ \\
Total & $27 / 293(9)$ & $151 / 347(44)$ & $93 / 188(49)$ & $125 / 283(44)$ \\
\hline
\end{tabular}

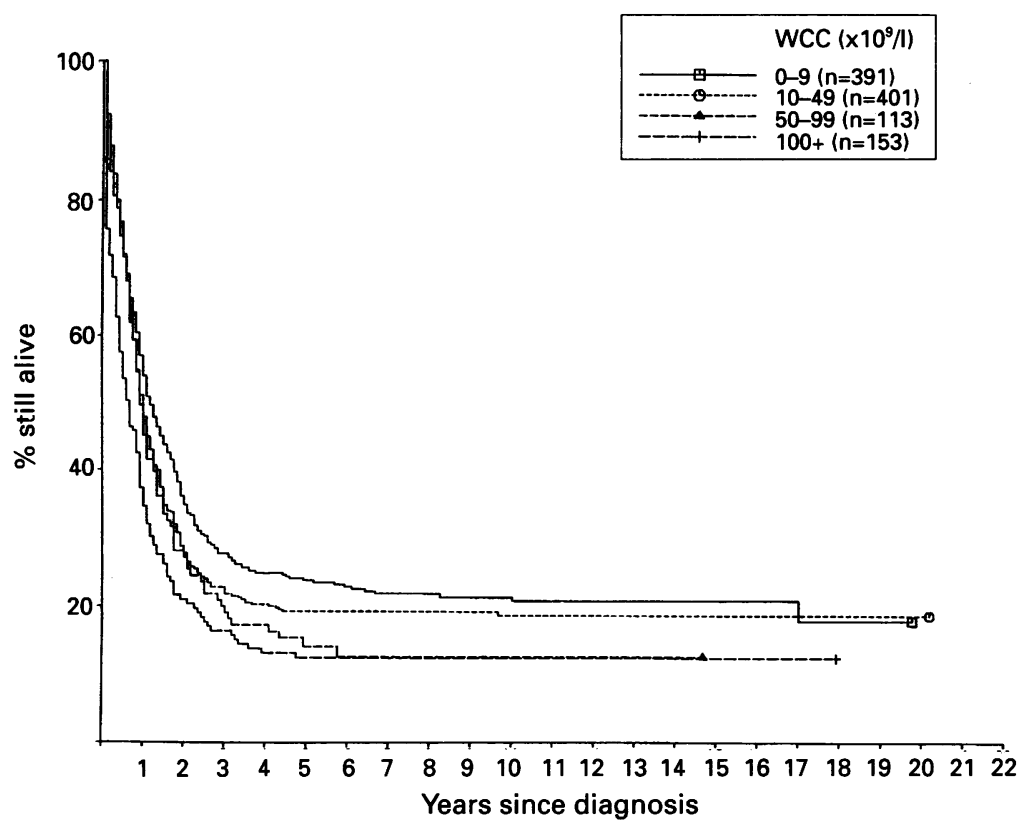

Figure 3 Actuarial survival curves for children first treated during the period 1971-88 classified by white cell count (WCC).

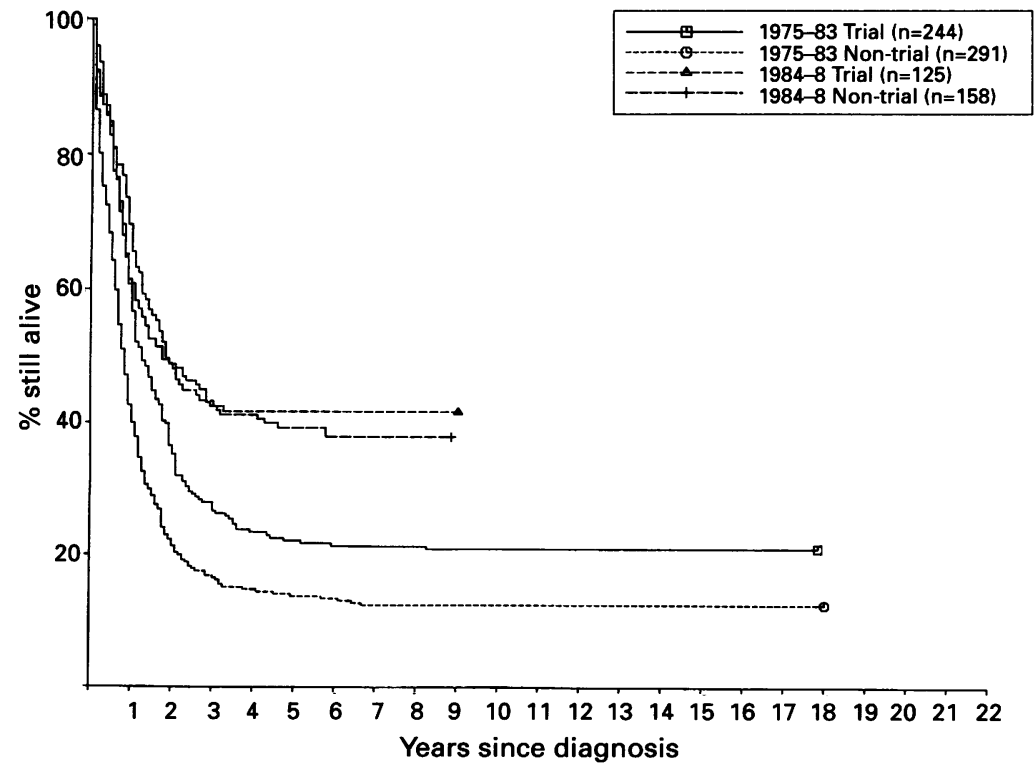

Figure 4 Actuarial survival curves for children first treated during the period 1975-88 classified by whether they were entered into national trials. trend in survival with white cell count was still statistically significant $\left(\chi^{2}=10.7\right.$ on $1 \mathrm{df}$, $p<0.01$ ), but the effects of age and FAB subtype were not.

The proportion of children treated at teaching hospitals increased throughout the study period, with the largest increase being between 1971-4 and 1975-9 (table 3). There was little variation with age in the proportion of children treated at teaching hospitals. Table 4 shows the trends in the proportions of children included in national trials. Few children were included in trials before 1975, the first year of entry to the childhood UKAML trial. Around $46 \%$ of children first treated in the period 1975-83, while UKAML and AML8 were open, were entered into trials. The proportion entered decreased slightly in 1984-8, but at least one major centre treated nearly all its patients in a local study with a standard protocol during this period. ${ }^{11}$ During 1975-88, 53\% of children aged 2-12 years when first treated were entered in trials, compared with $34 \%$ of those aged under 2 years and $29 \%$ of those aged 13-14 years. In all periods children treated at teaching hospitals were more likely to be entered into trials than those who were treated elsewhere.

Children entered in trials had a higher survival rate than non-trial children (fig 4: $x^{2}=8.84$ on $1 \mathrm{df}, \mathrm{p}<0.01$, allowing for age). Children treated at teaching hospitals had a higher survival rate than those treated elsewhere (fig 5: $\chi^{2}=5 \cdot 71$ on $1 \mathrm{df}, \mathrm{p}<0.05$, allowing for age). Among children treated at teaching hospitals in the period 1975-83, those who were entered into trials had a significantly higher survival rate than those who were not entered $\left(\chi^{2}=13.8\right.$ on $\left.1 \mathrm{df}, \mathrm{p}<0.001\right)$, but in the period 1984-8 there was no difference. At other hospitals the number of patients was too small to investigate the effect of trial entry on survival. When trial and non-trial children were analysed separately, there was no significant difference in survival between types of treatment centre for either group.

A total of $897(81 \%)$ children survived at least three months. In this group the trend towards higher survival in more recent periods of diagnosis was still highly significant $\left(\chi^{2}=90.5\right.$ on $\left.1 \mathrm{df}, \mathrm{p}<0 \cdot 0001\right)$. Survival rates were similar for boys and girls, and for Down's syndrome and non-Down's syndrome children. The lower survival rate for those aged less than 1 year at diagnosis just failed to attain statistical significance $\left(\chi^{2}=3 \cdot 81\right.$ on $\left.1 \mathrm{df}\right)$ but there were only 49 infants among the three month survivors. The effect of white cell count was still highly significant $\left(\chi^{2}=10.9\right.$ on $1 \mathrm{df}$ for trend, $\mathrm{p}<0.01)$. The FAB subtype had no effect on survival. Children entered in trials had a higher survival rate than those who were not, though not significantly so $\left(\chi^{2}=3 \cdot 17\right.$ on $1 \mathrm{df}$ allowing for age). There was also no significant effect of type of treatment centre $\left(\chi^{2}=1 \cdot 28\right.$ on $1 \mathrm{df}$ allowing for age).

Among the 535 (48\%) children who survived at least one year, the trend in survival rates with period of diagnosis was still highly significant $\chi^{2}=72.8$ on $\left.1 \mathrm{df}, \mathrm{p}<0.0001\right)$. There was no 


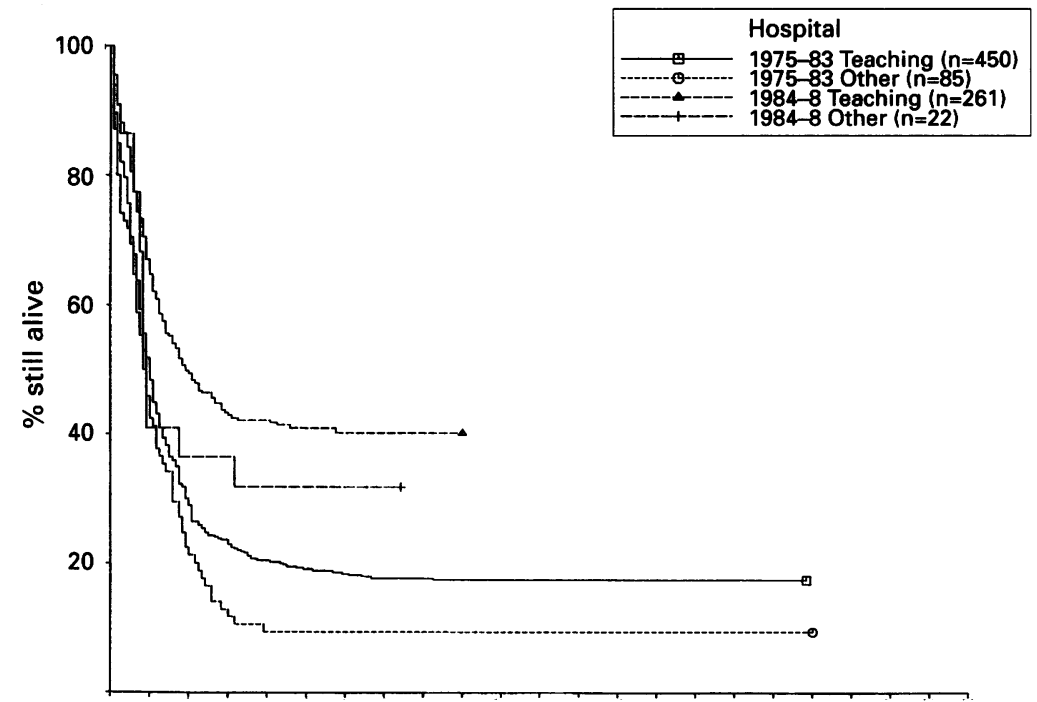

$\begin{array}{llllllllllllllllllllll}1 & 2 & 3 & 4 & 5 & 6 & 7 & 8 & 9 & 10 & 11 & 12 & 13 & 14 & 15 & 16 & 17 & 18 & 19 & 20 & 21 & 22\end{array}$

Years since diagnosis

Figure 5 Actuarial survival curves for children first treated during the period 1975-88 classified by type of treatment centre.

significant variation in subsequent survival with sex, presence of Down's syndrome, age, white cell count, FAB subtype, entry to trials, or type of treatment centre.

Most deaths of children with ANLL occurred within two years of diagnosis, but 123 $(37 \%)$ of the 334 two year survivors have so far been notified as dying subsequently. Of these, $65(53 \%)$ were known to have had a first relapse less than two years after diagnosis, 41 $(33 \%)$ had a first relapse later than two years after diagnosis (maximum five years), and eight $(7 \%)$ were in first remission from ANLL at the time of death; information on relapses was not available for the remaining nine (7\%) patients. Four of the eight deaths in first remission were due to doxorubicin hydrochloride (Adriamycin) related cardiomyopathy between 29 months and 10 years after the diagnosis of ANLL. Of the other four deaths, one was due to Pneumocystis carinii pneumonia and one to pulmonary fibrosis after bone marrow transplantation. One child, who also had Down's syndrome, died from complications of an operation for an atrial septal defect. The death of the remaining survivor was the only one so far recorded as being due to a second primary neoplasm; this patient had an adenocarcinoma of the ileum diagnosed 16 years after ANLL.

\section{Discussion}

The children analysed here constitute the largest population based series of children with ANLL to have been studied anywhere. The only other series we could find with a comparable number of patients consisted of 1006 children included in four successive therapeutic studies of the North American CCSG in the period 1972-83.12 The effects of several prognostic factors in the present study were broadly similar to those in the CCSG series. In the two series there was no difference in survival between the sexes. Infants aged less than 1 year had a significantly worse survival rate which was entirely due to higher mortality during the first year after diagnosis in the two studies. There was a highly significant trend of lower survival rates with increasing white cell count; in our study this was also confined to the first year after diagnosis.

The analysis of survival rates by $F A B$ classification was limited by several factors. Firstly, the subtype often could not be reliably deduced from the registry records, particularly for children diagnosed before 1980; before that date the distinction between type M1 and M2 leukaemia could rarely be made and M7 megakaryocytic leukaemia was not reliably identified. Secondly, the classifications are those reported by individual haematologists responsible for diagnosis. Interobserver variation in assigning subtypes is well documented, especially in distinguishing between $M 1$ and $M 2$ and in diagnosing M4, though agreement on classifying M5 (monocytic) leukaemia is greater. ${ }^{13}$ This subtype has been reported as occurring in a relatively high proportion of infants and young children ${ }^{14}{ }^{15}$; in the present study, $33 \%$ of children aged 0-2 years and first treated in the period 1980-8 were recorded as being of type $M 5$, compared with $11 \%$ of older children. M5 morphology has sometimes been reported as being associated with a lower survival rate ${ }^{15} 16$ but this was not so in our study. Patients with M3 (promyelocytic) leukaemia have been reported to have a good long term prognosis if they achieve remission. Among children in the present study who survived at least three months, however, there was little difference in subsequent survival rates between those with M3 leukaemia and those with other morphological subtypes. A higher survival rate for M2 leukaemia has been reported, ${ }^{17}$ but again we found little evidence for this. Other investigators have also found no significant effect of morphology, including the CCSG study which was based on large numbers of children. ${ }^{12} 18$

Children with Down's syndrome who were treated for ANLL had a similar survival rate to non-Down's syndrome children. This result is in agreement with a previous report, ${ }^{19}$ but many patients in the present series were also in that earlier study. It appears that, unlike in acute lymphoblastic leukaemia, the presence of Down's syndrome is not an unfavourable prognostic factor for childhood ANLL. A higher proportion of Down's syndrome children than non -Down's syndrome children did not receive any treatment for their leukaemia, however, even in the most recent calendar period. The children with Down's syndrome who were not treated may have been weighted towards those with an especially poor prognosis, as the proportions untreated were particularly high among infants and among children who also had other congenital abnormalities. Nevertheless, the results of this study, with larger numbers of patients and longer follow up, tend to confirm the earlier suggestion that Down's syndrome in itself does not imply a worse prognosis in childhood ANLL. Among children with ANLL diagnosed in the five Nordic 
countries in 1984-91, those with Down's syndrome had a more favourable prognosis. ${ }^{20}$

Few prognostic factors have been consistently identified in childhood ANLL. Refined classification based on immunology and cytogenetics in addition to morphology may reveal subgroups of patients with an especially good or poor prognosis. Prognostic factors probably also depend strongly on the quality and intensity of treatment and may well change as new protocols are introduced. The high probability of subsequent survival among patients who have already survived one year emphasises the importance of achieving first remission in this disease.

Half of the deaths in first remission so far observed among the two year survivors in this series have been due to Adriamycin related cardiomyopathy. This is an important complication of anthracycline chemotherapy for a wide range of childhood cancers and survivors are at risk for at least 20 years. $^{21}$

The dramatic increase in survival rates for childhood ANLL between 1971 and 1985 has previously been reported. ${ }^{1}$ This study shows that the higher survival rates among children diagnosed during the 1980 s have been maintained during a longer period of follow up, and that the improvement in prognosis for newly diagnosed children has continued to 1988 . As there has been little change in the proportion of children not receiving any treatment for their leukaemia, the improved survival is entirely due to more effective treatment.

In a previous study of children with ANLL first treated in the period 1977-84 and with relatively short follow up, those who were treated at a teaching hospital had a higher survival rate. ${ }^{9}$ This result is confirmed here, using data from a longer period and with longer follow up. The effect of entry to clinical trials on the survival rate has not previously been studied for childhood ANLL. The finding of a higher survival rate for children in trials is similar to previous results relating to acute lymphoblastic leukaemia. ${ }^{8}$ The absence of significant effects of type of hospital or entry to trials on the subsequent survival rates of three month survivors may be due to selective nonreferral to teaching hospitals and non-entry into trials of children with a particularly poor prognosis, but it should be emphasised that untreated children were excluded from all the survival analyses and that high mortality in the first months after diagnosis resulted in relatively small numbers of children being included in the analyses of longer term survival. For cases of childhood acute lymphoblastic leukaemia first treated in Britain in the period 1971-84, the size of treatment centre had a significant effect on survival of non-trial patients. No such effect was found in this study, but this could be due to the fact that only small numbers of children were treated at nonteaching hospitals in more recent years, while in earlier years there were fewer survivors overall.

We thank the many consultants and general practitioners who provided the information on which this paper is based. We are provided the information on which this paper is based. We are grateful to the Office of Population Censuses and Surveys, the Information and Statistics Division of the Common Services Agency of the Scottish Health Service, the Registrar General for
Scotland, regional cancer registries, the Clinical Trial Service Scotland, regional cancer registries, the Clinical Trial Service Unit and the UKCCSG for providing copies of notifications of childhood leukaemia cases and also to the National Health Service Central Registers at Southport and Edinburgh for notification of deaths and the flagging of survivors. We are grateful to Mr M Loach for help with computing, to Mrs M Allen for her part in collecting the medical records, and to Mrs E M Roberts for secretarial help. The Childhood Cancer Research Group is supported by the Department of Health and the Scottish Home and Health Department.

1 Stiller CA, Bunch KJ. Trends in survival for childhood cancer in Britain diagnosed 1971-85. Br f Cancer 1990 62: $806-15$.

2 Medical Research Council's Working Party on Leukaemia in Adults. Treatment of acute myeloid leukaemia with daunorubicin, cytosine arabinoside, mercaptopurine, L-asparaginase, prednisone and thioguanine: results of treatment with five multiple-drug schedules $\mathrm{Br} F$ Haematol 1974; 27: 373-89.

3 Medical Research Council. Immunotherapy of acute myeloid leukaemia. Br $\mathcal{F}$ Cancer 1978; 37: 1-14.

4 Medical Research Council. Chemotherapy of acute myeloid leukaemia in adults. $B r F$ Cancer 1979; 39: 68-86.

5 Chessells JM, Sieff CA, Rankin A. Acute myeloid leukaemia in childhood: treatment in the United Kingdom. Haematology and Blood Transfusion 1983; 28: 51-5.

6 Rees JKH, Gray RG, Swirsky D, Hayhoe FGJ. Principal results of the Medical Research Council's 8th acute myeloid leukaemia trial. Lancet 1986; iv: 1236-41.

7 Marcus RE, Catovsky D, Prentice HG, et al. Intensive induction and consolidation chemotherapy for adults and children with acute myeloid leukaemia (AMI). Join AML trial 1982-85. Haematology and Blood Transfusion 1987; 30: 346-51.

8 Stiller CA, Draper GJ. Treatment centre size, entry to trials and survival in acute lymphoblastic leukaemia. Arch Dis Child 1989; 64: 657-61.

9 Stiller CA. Centralisation of treatment and survival rates for cancer. Arch Dis Child 1988; 63: 23-30.

10 Bennett JM, Catovsky D, Daniel MT, et al. Proposals for the classification of the acute leukaemias. Br $\mathcal{F}$ Haemato 1976; 33: 451-8.

11 Sartori PCE, Taylor MH, Stevens MCG, Darbyshire PJ Mann JR. Treatment of childhood acute myeloid leukaemia using the BFM-83 protocol. Med Pediatr Oncol 1993; 21: 8-13.

12 Buckley JD, Chard RL, Baehner RL, et al. Improvements in outcome for children with acute nonlymphocytic leukemia. A report from the Children's Cancer Study Group. Cancer 1989; 63: 1457-65.

13 Argyle JC, Benjamin DR, Lampkin B, Hammond D. Acute nonlymphocytic leukemias of childhood. Inter-observer variability and problems in the use of the FAB classification. Cancer 1989; 63: 295-301.

14 Darbyshire PJ, Smith JHF, Oakhill A, Mott MG Monocytic leukemia in infancy. A review of eight children. Cancer 1985; 56: 1584-9.

15 Creutzig U, Ritter J, Riehm H, et al. Improved treatment results in childhood acute myelogenous leukemia: a report 1985; 65: 298-304.

16 Chessells JM, O'Callaghan U, Hardisty RM. Acute myeloid leukaemia in childhood: clinical features and prognosis. Br f Haematol 1986; 63: 555-64.

17 Creutzig U, Ritter J, Schellong G. Identification of two risk groups in childhood acute myelogenous leukemia after therapy intensification in study AML-BFM-83 as compared with study AML-BFM-78. Blood 1990; 75: 1932-40.

18 Phillips M, Richards S, Chessells J. Acute myeloid leukaemia in childhood: the costs and benefits of intensive treatment. Br f Haematol 1991; 77: 473-7.

19 Levitt GA, Stiller CA, Chessells JM. Prognosis of Down's syndrome with acute leukaemia. Arch Dis Child 1990; 65: 212-6.

20 Lie SO, Gustafsson G, Jonmundsson G, Mellander I Siimes MA, Yssing M. A population based study of children with AML in the five Nordic countries. children with AML in the five Nordic countries. Treatment results and risk factors before and after intensi-

21 Steinherz LJ, Steinherz PG, Tan CT, Heller G, Murphy L. Cardiac toxicity 4 to 20 years after completing anthracycline therapy. $\mathscr{f} A M A$ 1991; 266: 1672-7. 